\title{
Virtual reality for automotive radars
}

\author{
M. E. Gadringer, H. Schreiber, A. Gruber, M. Vorderderfler, D. Amschl, W. Bösch, S. Metzner, H. Pflügl, \\ M. Paulweber
}

\begin{abstract}
Car manufacturers spend quite a lot on the development of driver assistance systems and subsequently on autonomous driving functionality. To ensure the safety and reliability of these functions meet industrial standards it is necessary to verify and validate their functionality. While tests on the road are still the ultimate evidence of correct operation they are associated with huge efforts and risks. Therefore, they have to be complemented by other means like simulations and tests on specialised testbeds. For the latter the car's sensors have to be stimulated in a way that they perceive a desired - but only virtual - environment. An important type of sensor in cars is the radar due to its various advantages. This article describes the development of a stimulator generating virtual radar targets in order to enable the testing of autonomous driving functions.
\end{abstract}

Keywords: radar systems; test equipment; radar applications; radar equipment; radar signal processing; radio frequency circuit design

\section{Virtuelle Realität für Radargeräte in Autos.}

Autohersteller investieren eine hohe Summe in die Entwicklung von Fahrerassistenzsystemen und in autonomes Fahren. Es ist daher notwendig, die sichere und zuverlässige Funktion dieser Systeme zu verifizieren und zu validieren. Dies kann mit Testfahrten auf der Straße erfolgen, was aber mit großen Kosten und hohem Aufwand verbunden ist. Es wird daher daran gearbeitet, zumindest Teile dieser notwendigen Tests mit Simulationen auf Prüfständen durchzuführen. Das Radar ist ein wichtiger Sensor für die erwähnten Systeme, deshalb ist dessen Stimulation von entscheidender Bedeutung. Dieser Beitrag beschreibt die Entwicklung eines Stimulators zur Erzeugung von virtuellen Radarzielen, um autonome Fahrfunktionen auf dem Prüfstand zu testen.

Schlüsselwörter: Radarsysteme; Testsysteme; Radaranwendungen; Radartestgeräte; Radarsignalverarbeitung; Hochfrequenzschaltungsentwurf

\section{Introduction}

Multiple different advanced driver assistance systems (ADAS) are implemented in modern vehicles. These systems need sensors in order to perceive the vehicles environment including other traffic like cars, cyclists or pedestrians and static objects (i.e.: the road itself, obstacles, etc.). Currently, the most used sensors for these applications are ultrasonic sensors, cameras, lidars and radars [1]. As enabler for autonomous driving functions (ADF), ADAS systems will become even more important. But before autonomous driving can become broadly accepted, the ADAS functions have to be verified and validated. The traditional way to accomplish this goal is testing the functionality by driving either on proving grounds or on public roads. Safety experts assume that distances between 1 and 10 million $\mathrm{km}$ [2], [3], [4] are necessary to guarantee a risk less than or equal to human drivers. One drawback of this approach is, that it is difficult or impossible to exactly reproduce situations which endanger other road users. But especially these extreme situations are of interest and need to be repeated until the ADFs operate in a satisfactory manner. To reduce the danger and the cost of these tests, other supporting verification and validation ( $V \& V$ ) methods have to be or have already been introduced. One possibility is the software simulation of parts or the whole system. Another one are hardware-in-the-loop (HiL) tests of components. But in the end the whole vehicle with all its systems has to be tested. In order to reduce the beforehand mentioned testing kilometres on roads even further vehicle-in-the-loop (ViL) tests on specialised testbeds are envisaged. Since the ADF systems interact with the environment by their sensors it is necessary that these sensors "see" the intended scenario consisting of other cars, pedestrians and obstacles. Such a test environment is already arranged using a large hall with remotely controlled vehicles and dummies [5], [6], [7]. But while this addresses the issues danger and repeatability it is still a huge effort both in time - the scenarios have to prepared - and expenses. Therefore, further efforts are undertaken to provide the sensors with "virtual reality goggles" meaning that the environment exists only virtually in software but physical stimuli are given to the sensors in order to let the sensors think that this scenario is real. Such an approach allows V\&V tests to be performed on testbeds and is the idea behind the DrivingCube тм by AVL List GmbH (see Fig. 1) [9].

In this paper we introduce the concepts to stimulate one kind of sensor, namely the automotive radar. A short overview of automotive radar and its stimulation are given in Sect. 2.1 and 2.2, respectively. A radar target stimulator (RTS) has been developed th the

Gadringer, Michael E., Institute of Microwave and Photonic Engineering, Graz University of Technology, Inffeldgasse 12, $8010 \mathrm{Graz}$, Austria

(E-mail: michael.gadringer@tugraz.at); Schreiber, Helmut, Institute of Microwave and Photonic Engineering, Graz University of Technology, Inffeldgasse 12, 8010 Graz, Austria; Gruber, Andreas, Institute of Microwave and Photonic Engineering, Graz University of Technology, Inffeldgasse 12, $8010 \mathrm{Graz}$, Austria; Vorderderfler, Michael, Institute of Microwave and Photonic Engineering, Graz University of Technology, Inffeldgasse 12, 8010 Graz, Austria; Amschl, Dominik, Institute of Microwave and Photonic Engineering, Graz University of Technology, Inffeldgasse 12, 8010 Graz, Austria; Bösch, Wolfgang, Institute of Microwave and Photonic Engineering, Graz University of Technology, Inffeldgasse 12, 8010 Graz, Austria; Metzner, Steffen, AVL List GmbH., Hans-List-Platz 1, 8020 Graz, Austria; Pflügl, Horst, AVL List GmbH., Hans-List-Platz 1, 8020 Graz, Austria; Paulweber, Michael, AVL List GmbH., Hans-List-Platz 1, 8020 Graz, Austria 


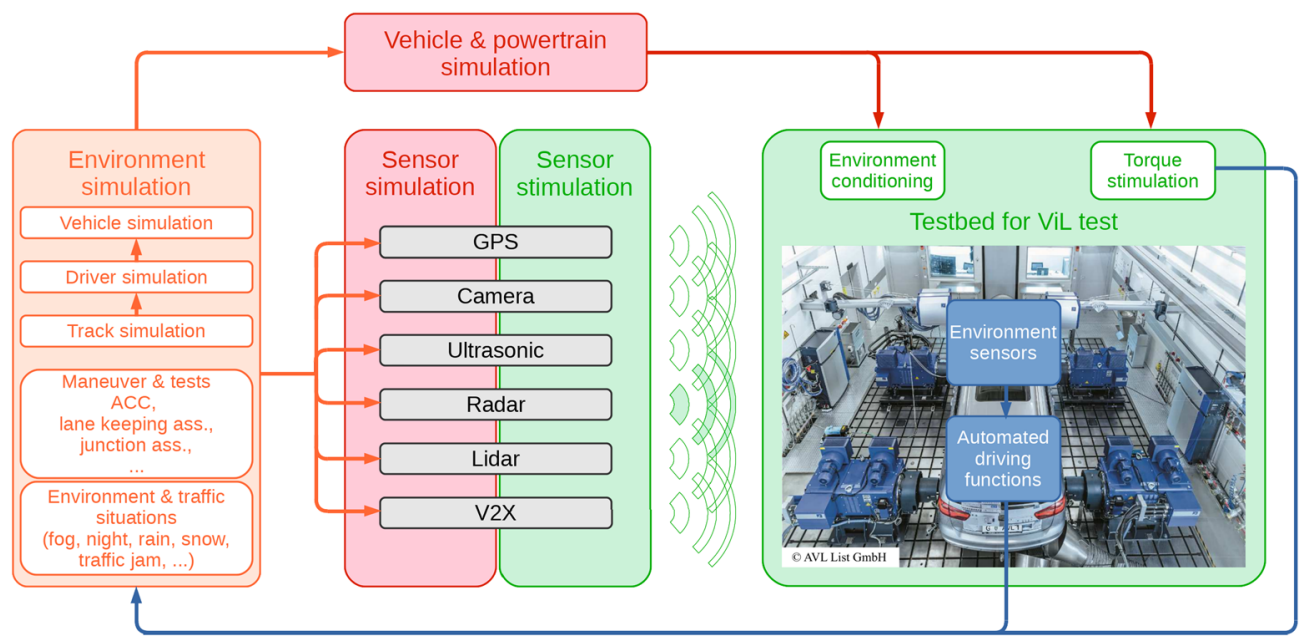

Fig. 1. Sensor testing embedded in a Driving-Cube ${ }^{\mathrm{TM}}$ test bench [8], [9]

Institute of Microwave and Photonics Engineering of Graz University of Technology in corporation with AVL List GmbH. The software necessary for generating the input parameters for the RTS was derived by the Institute of Automation and Control (also Graz University of Technology). The design considerations and the components of this RTS are presented in Sect. 3 in detail. Section 5 provides a short outlook on the future of automotive radar sensors and the impact of these trends on target stimulation. Thereafter, we present a summary on this paper in Sect. 6 .

\section{Automotive radars and its stimulation}

\subsection{Principles of automotive radar}

Radar has a unique position among the automotive sensors responsible for the perception of the environment. Compared to e.g. ultrasonic sensors it works both in the near- \& far-range. Due to its flexibility the radar sensor is used in connection with multiple ADFs (like automatic cruise control, emergency breaking or blind spot detection) [10]. Based on the active and coherent mode of operation of the radar (i.e.: the sensor creates its own target illumination), it is also able measuring the relative velocity of other objects. Furthermore, radar sensors work reliably also under adverse weather conditions like fog and rain where optical systems (e.g. cameras or lidars) have difficulties [11].

Automotive radar sensors have achieved maturity since they have been used for quite some years, especially in high-end cars. Moreover, the underlying technology has been around in many other radar applications before. Since its introduction, the automotive radar has undergone a significant evolution. The operating frequency has changed from $24 \mathrm{GHz}$ to $77 \mathrm{GHz}$. This shift to higher centre frequencies was, on the one hand, initiated by legislative regulations. On the other hand, higher centre frequencies allow higher bandwidths of the transmit signal. Frequencies even above $100 \mathrm{GHz}$ are envisaged for the future [12]. Furthermore, the waveform of the radar's transmit signal changed. While in the beginning the classical pulsed system was used, today the frequency modulated continuous wave (FMCW) principle with fast chirp sequences is primarily utilised. Similar to other radars there were also significant evolutions in the antenna design. At the beginning the antennas were mainly steered mechanically in the azimuth direction. The current and future generations of automotive radars are based on arrays of patch antennas allowing electronic beam steering or digital beam forming not only in azimuth but also in elevation.

\subsection{Basics of radar target stimulation}

To stimulate radars in general and automotive radar in particular, the transmitted signal of the radar has to be changed in a way that represents one or more target objects and sent back to their radar receiver. The radar will then "see" those virtual - not existing in reality - targets in the same way as it would detect real objects ("deceptive jamming", [13], [14], [15]). There are 4 target parameters which are important for the detection process in the radar:

- distance between the radar ("ego-vehicle") and the target: target range $R$,

- direction to the target given by the azimuth and elevation angles,

- relative speed between the ego-vehicle and the target, but only the radial velocity component $v$ (range-rate) is relevant,

- radar cross section (RCS) of the target which defines the amount of energy reflected back to the radar.

Range, velocity and RCS will influence the echo signal directly and the RTS has to modify the signal accordingly:

- delay the echo by the amount $\tau$

$$
\tau=\frac{2 R}{c_{0}},
$$

- shift the frequency by the Doppler frequency $f_{D}$, where $f_{0}$ is the radar centre frequency and $c_{0}$ is the velocity of light

$$
f_{D}=\frac{2 v f_{0}}{c_{0}}
$$

- attenuate or amplify the signal by a gain $G$ depending on the RCS $\sigma$ and the distance

$$
G \propto \frac{\sigma}{R^{4}} .
$$

Some implementations of a RTS directly inject the necessary echo signal at the RF port of the radar sensor. In this way, they forgo the free-space wave propagation and the radar's antenna altogether. Such an approach is unfavourable at a ViL testbed due to two reasons. On the one side, the direct connection of the RF port requires modification at the vehicle and the sensor itself. Such interventions should be kept to an absolute minimum on a testbed. On the other 
side, the impact of parasitic reflections caused by the bumper or other part of the vehicle's body are neglected. Hence, the behaviour of the sensor will differ from the one recognised in road tests. Therefore, the stimulation has to be accomplished using the original interface of the radar - the antenna.

In order to stimulate different target angles, the RTS needs a way to change the direction from which the radar receives the echo signal. This can be accomplished by either moving one or more transmit antennas of the RTS. Another possibility is applying multiple parallel transmit channels where each channel is responsible for one direction. An alternatively approach, the rotation of the radar sensor itself, is not feasible for ViL tests as it requires a massive intervention on the vehicle. Additionally, this solution supports only a single independently moving target.

\section{Implementation of the RTS system}

\subsection{Requirements}

A ViL-test equipment for ADAS functions has different requirements for a RTS than a test system for verifying the radar sensor itself. The latter has to prove that the sensor is working correctly and validates its performance or electrical parameters. In general this can be accomplished with a single target. Only for testing the radar's resolution - in range, angle and velocity - a second target is necessary. In the radar's so-called target mode - no tracking algorithms are used to generate the output - it is not even necessary to change the target's distance even if the target shows a Doppler-shift. The radar's range and velocity accuracy can be tested independently.

In contrast, a RTS-system for ADAS V\&V has to generate the radar echos of complex and realistic traffic scenarios which are often generated by environment simulation systems (e.g. [16]). Those virtual targets have to move independently. To ensure that the radar's tracking algorithm works as desired the stimulated velocity must be equal to the change rate in distance. Otherwise, the radar processor will ignore the target due to its physically impossible attributes.

As mentioned in Sect. 2.2 radar target stimulation on a ViL testbed applies Over-the-air (OTA) injection of the echo signals via the radar's antennas. Unfortunately, OTA stimulation will introduce another problem: reflections from the surrounding environment which is called clutter. Unlike in reality, where the static environment has a Doppler-shift in regard to the moving radar, on the testbed both, the radar and the environment, are static resulting in zero Doppler-shift. It is difficult for the radar-processor to cope with such non-standard circumstances. For a proper stimulation on a testbed any clutter must be eliminated as much as possible.

Since a ViL testbed should be usable for different vehicles and, therefore, also different radar sensors the design of the RTS shall not impose any constraints on the radar, its design and its waveform. At best, the RTS should successfully stimulate the sensor without any a-priori knowledge about the radar.

Additionally, the intended application for the RTS described in this article imposes another requirement which is difficult to achieve: the focus of the RTS should be on the stimulation of targets at short distances (down to about 2 metres). Nevertheless, it has also to cover the radar's instrumented range which is typically about $250 \mathrm{~m}$. Furthermore, todays automotive radar have a bandwidth up to $1 \mathrm{GHz}$ resulting in a range resolution of $15 \mathrm{~cm}$. The resolution of the RTS has to be better than that.

The implications of these requirements on the design of our RTS are described in Sect. 3.2.

\subsection{Concept and general design}

In the framework of this article only the hardware of the RTS is described. The parameters necessary to stimulate the targets are prepared by the RASIG software which are provided as digital data [17].

The concept and design of our RTS was strongly influenced by the requirements given in Sect. 3.1. Especially, the low minimum distance of $2 \mathrm{~m}$ (corresponding to a time delay of $13 \mathrm{~ns}$ ) together with the fact that we have to support a wide range of radar sensors (no a-priori knowledge about the radar's waveform) forces an analogue implementation. Without a recording and playback mechanism - which is not possible if the radar transmit signals change such low latencies cannot be achieved in the digital domain.

Unfortunately, the complexity of analogue based RTS increases more or less linearly with the maximum target range and the number of targets to be stimulated. The above given maximum range and delay line resolution would result in nearly 1700 delay sections which is clearly not feasible. To lower the complexity, a hybrid solution consisting of both an analogue and a digital system is used to cover the whole range of an automotive radar $(2-250 \mathrm{~m})$. The hand-over distance between the two subsystems is set to $30 \mathrm{~m}$. This is the shortest delay realisable by state-of-the-art digital processing modules consisting of an analogue-digital-converter (ADC), a digital-analogue-converter (ADC) and a field programmable gate array (FPGA) in between (see Sect. 3.7).

Although $30 \mathrm{~m}$ can be achieved with acceptable effort, we decided on a concept for the analogue RTS which offers a good scalability, both in the distance and the number of targets. In this way, the RTS can be optimised for the intended test cases. This scalable concept is shown in Fig. 2.

To accommodate the different radar frequencies we decided on processing the radar signal at an intermediate frequency (IF). In this case the RTS can be adapted for different radar centre frequencies by changing the frontend only. Furthermore, the full implementation of the RTS at $77 \mathrm{GHz}$ is of much higher complexity compared to frequencies below $10 \mathrm{GHz}$. In the end, an IF of $2 \mathrm{GHz}$ was chosen due to the availability of suitable components which support a bandwidth of $1 \mathrm{GHz}$ (most state-of-the-art long-range-radars use a signal bandwidth of up to $1 \mathrm{GHz}$ ). Furthermore, the selected IF frequency works well with the ADC and the DAC of the digital RTS module.

The components of the analogue RTS responsible for modifying the signal parameters (as described in Sect. 2.2) are presented in the following sections.

\subsection{Delay line module}

Variable delays are normally implemented by binary switched delay lines because this method scales good in the range dimension (doubling the range needs only one additional delay segment). But it scales badly in regard to the number of targets. Since such a delay line cannot generate delays for multiple independent targets each target would need its own delay line. An alternative approach is the so-called tapped delay-line. It consists of delay segments of identical length corresponding to the minimum desired delay. The number of these segments increases linearly with the maximum range but each possible delay can be achieved simultaneously by using couplers between the segments. This concept is presented in Fig. 3 and was chosen for our RTS. In order to reduce the number of necessary delay segments we chose a length of $60 \mathrm{~cm}$ for those (corresponding to $30 \mathrm{~cm}$ range resolution). Since the range accuracy was required to be better than this value an additional adjustment of the delay was implemented in the target emulation modules (TEM) as described in Sect. 3.5. This allocation reduced the number of switches and couplers significantly (e.g. the break even point regarding the number 


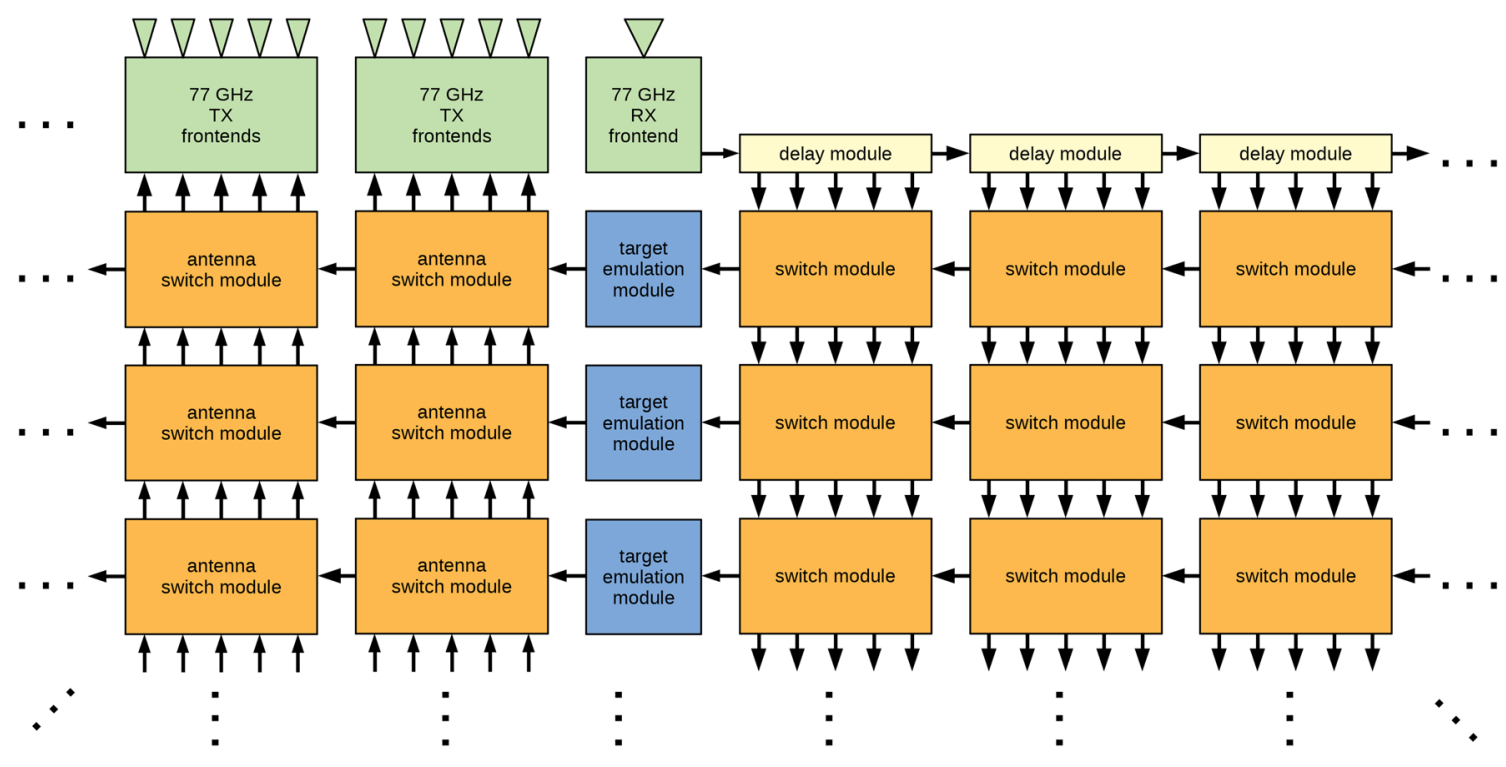

Fig. 2. Overall system concept of the analogue RTS

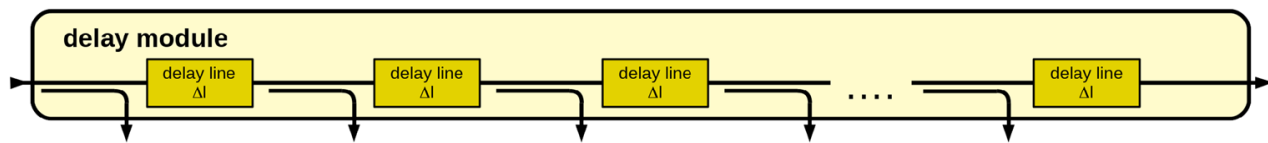

Fig. 3. Schema of a single delay line module

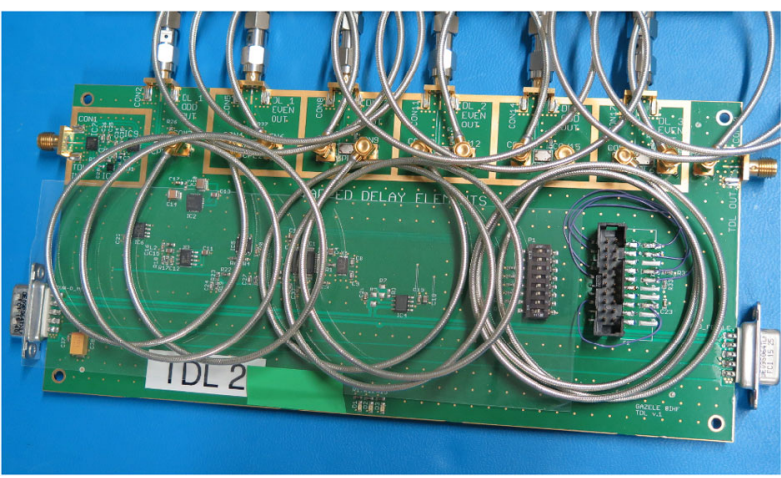

Fig. 4. Single delay line module

of switches between the chosen solution and a high-resolution delay line would be at 50 targets). A photo of the current delay line module with 6 segments and a delay of $360 \mathrm{~cm}$ - corresponding to a target range of $180 \mathrm{~cm}$ - is given in Fig. 4 .

For the first demonstrator the delays themselves were implemented by coaxial cables. In future designs this realisation will be changed to achieve better integration on printed circuit boards $(P C B)$. For example, defective-ground-structure transmission lines could be used.

\subsection{Switch matrix \& modules}

The switch modules form the interconnection between the delay modules (Sect. 3.3) and the TEMs (Sect. 3.5) as shown in Fig. 2. A target is moved by switching the input of its TEM from one tap of the delay line to the next. Since hot-switching from one delay to another will cause undesired phase jumps of the stimulator's output
- which does not happen in the real world - measures were taken to prohibit this: instead of a single connection to the TEM there are two, one can be connected to even-numbered delays the other one to odd-numbered ones (see Fig. 5). Using a blend-over mechanism in the TEM cold-switching between those two inputs is ensured.

The chosen design of the switch matrix does not only allow oneto-one routing of a delay line tap to a TEM but also feeding the same delay to multiple TEMs (one-to-many) and even different delays to the same TEM (many-to-one) enabling the generation of targets with a range extension covering multiple delay segments.

\subsection{Target emulation module}

The TEM is the component of the analogue RTS which adjusts the received radar signal in order to generate a virtual target with the desired velocity and RCS properties. Generally, one TEM can only provide a single target. Therefore, the numbers of targets which can be stimulated depends on the number of TEMs in the system. The block diagram of a TEM is provided in Fig. 6 .

As mentioned in Sect. 3.3 the TEM improves the range resolution of the stimulator by a factor of 4 by providing additional delay segments. The length of those sub-delays are a quarter of the original delay segments. Variable attenuators allow a blend over from one delay to another. This blend over allows to switch a delay segment on or off while the signal of this channel is not active. This principle is depicted in Fig. 7.

Since the target range is not changed at the radar frequency but by switching the delay at the much lower IF the phase change produced by this is also much lower than that of a target moving in reality (Doppler shift, see Eq. (2)). It is one of the tasks of the TEM to generate the correct Doppler frequency shift which enables the radar to detect the correct target velocity. This is accomplished by a vectormultiplier which is fed with the I/Q-components of the Doppler sig- 


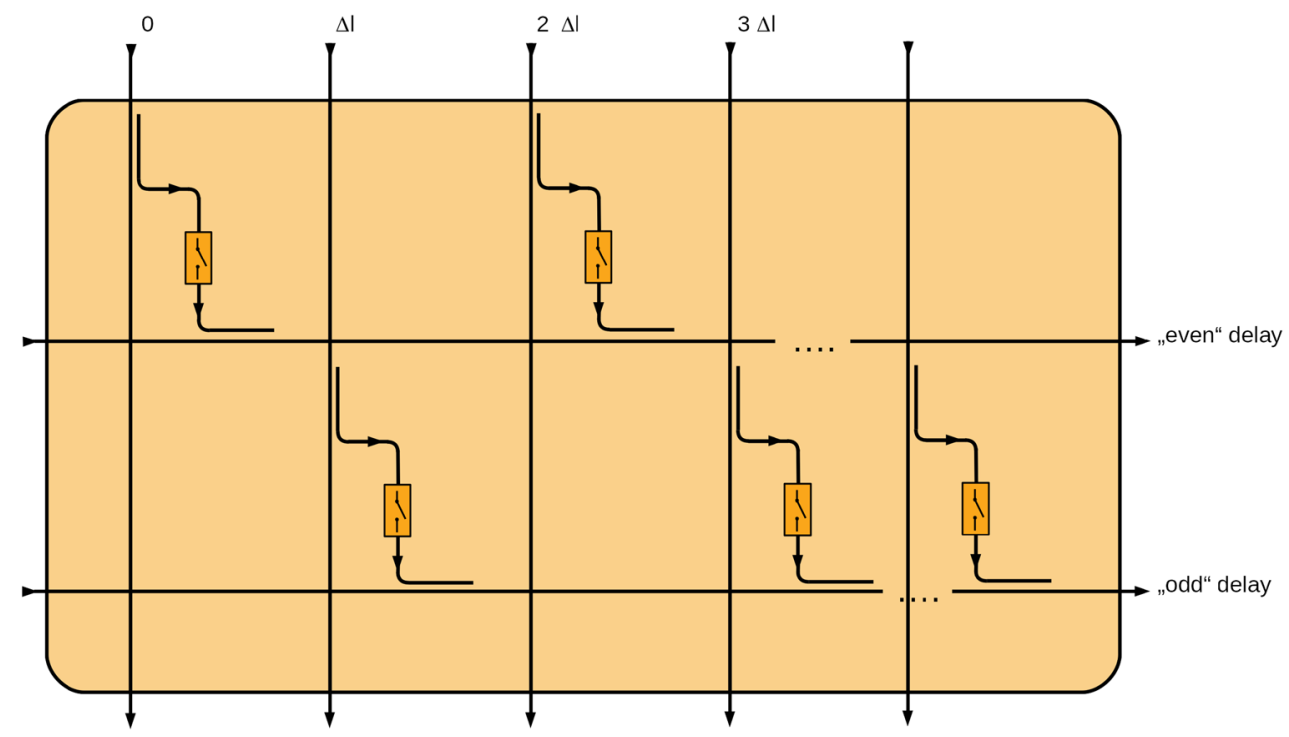

Fig. 5. Schema of a single switch module

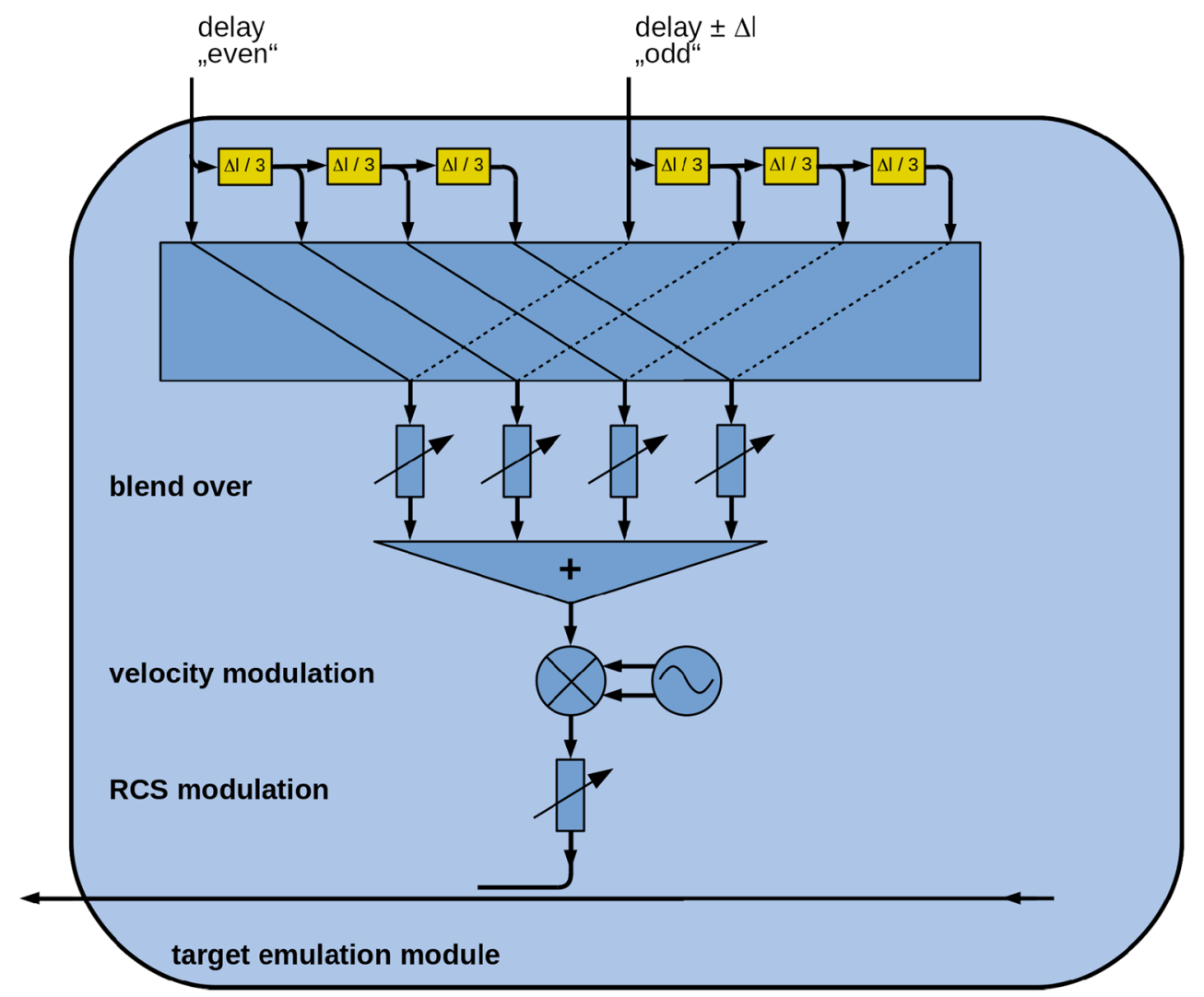

Fig. 6. Schema of a single TEM 


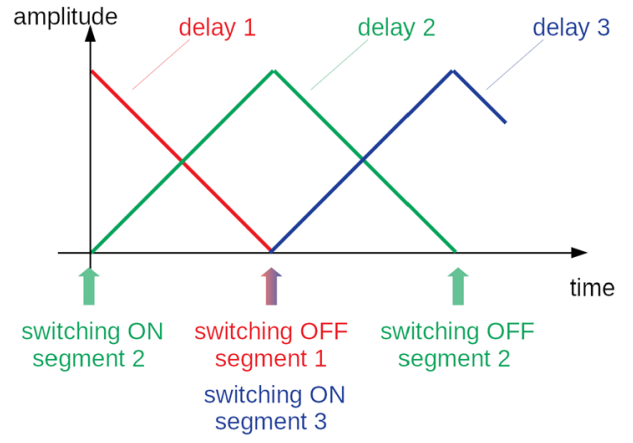

Fig. 7. Blend over principle

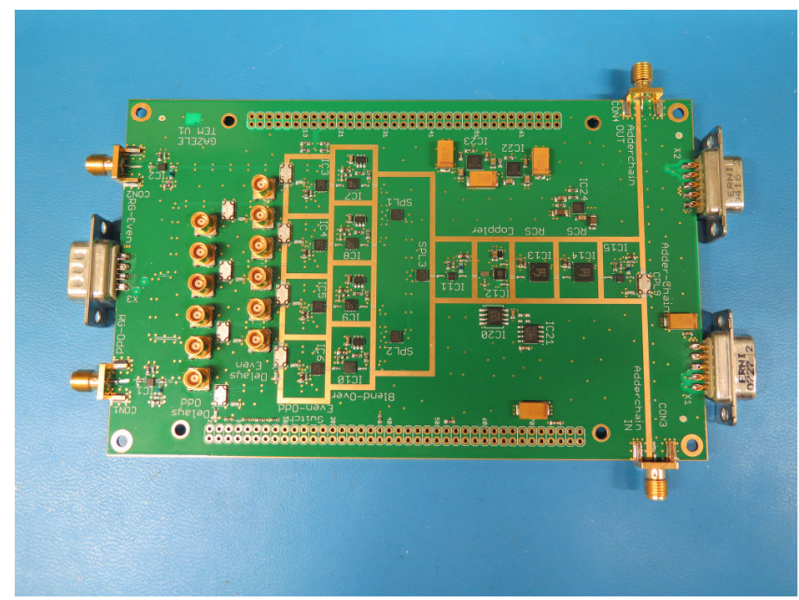

Fig. 8. Single delay TEM

nal. These Dopper signal components can be simple sine-waves in order to shift the echo-signal in frequency, or, a more complex signal providing also a spectral spread of the target echo. The Dopper signal components are generated by a digital synthesiser implemented on the control board of the TEM.

Furthermore, the TEM has to correctly set the amplitude. As shown in Eq. (3) this depends both on the target distance and its RCS. Especially the dynamic range due to distance variations can be quite large. E.g. the same object positioned at 3 or $300 \mathrm{~m}$ has a difference in the echo power of $80 \mathrm{~dB}$. To accomplish this task the TEM applies a cascade of several programmable attenuators. Figure 8 shows a photo of a TEM.

\subsection{Antenna switch module}

For angular target movement the echo signal must be switched between different transmit antennas. The chosen antenna determines the perceived target's azimuth.

A switch matrix with a similar concept as the delay switch matrix (Sect. 3.4) is used for routing the signal from a TEM to an antenna frontend. It has to support a one-to-many signal routing for lateral extended targets and many-to-one connections for multiple targets in the same direction.

\subsection{Digital RTS implementation}

As indicted in Sect. 3.2 a digital RTS is necessary to make the stimulation of long-range targets feasible. To set the hand-over range as low as possible we selected the cutting edge hardware platform AV104 from ApisSys [18] (Fig. 9). This module combines an ADC and

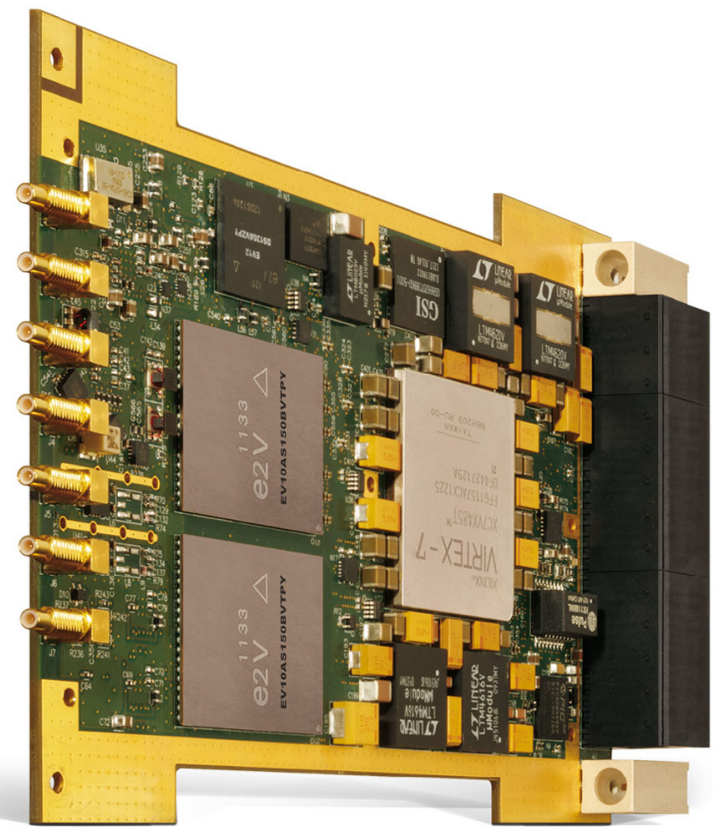

Fig. 9. ApisSys AV104 module (courtesy of ApisSys)

a DAC with extremely low latencies with a high capacity FPGA. The resulting overall minimum latency of $180 \mathrm{~ns}$ - one of the lowest latencies available for civil application - correlates to a target distance of $27 \mathrm{~m}$.

The digital RTS is capable to process signals with a bandwidth of up to $1 \mathrm{GHz}$ (more or less the same as the analogue RTS).

The firmware is based on a classical Digital Radio Frequency Memory (DRFM) structure. Massive parallel processing in the FPGA is necessary to ensure the required throughput. The frequency shift for each target is performed by a complex mixer fed by a numerically controlled oscillator. Afterwards, the delay is implemented independently for each target by a block RAM controlled by a programmable shift register. The resolution of the delay is about $5 \mathrm{~cm}$. Thereafter, the signal's power level is set by a multiplier. The FPGA should be able to stimulate up to 30 targets. In the last step the signals of all targets are combined and sent to the DAC which has to provide the necessary dynamic range.

The module is equipped with a single DAC which restricts the digital RTS to a single azimuth angle. In order to stimulate multiple directions either multiple boards or a new board with multiple DACs are necessary whose outputs are connected to the antenna switch matrix in the same way as the TEMs.

\section{Function tests}

The correct operation of the RTS has been validated both in the laboratory and on a complete roller test bench (see Fig. 10). The stimulator was able to create targets which were successfully detected and tracked by the radar sensor (see Fig. 11). Using both the analogue and digital modules the RTS was able to cover the whole required range.

Those tests have also been used to minimise and partly eliminate the stationary clutter from the testbed's surroundings. We were able to successfully suppress the clutter by the use of a few radar absorbing plates without impeding the artificial air flow to the vehicle too much. 


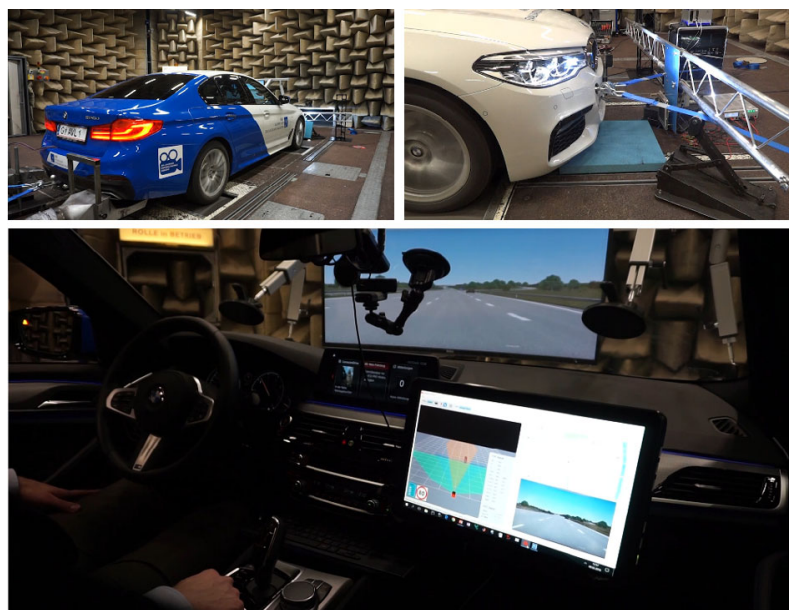

Fig. 10. Pictures from the testbed

\section{Outlook}

There are already a few indications how automotive radars will evolve in the future. As indicated in Sect. 2.1 the next generations of radar sensor will move to even higher frequencies. This can be easily covered with our RTS by changing the RF-frontend. More of a problem will be introduced, if the bandwidth of the radar signal is significantly increased as well.

Another trend in the development of upcoming radars is to combine radar sensor functionality with additional features, like communication. It would be no problem to add such functionality to the radar by using an appropriate modulation scheme like orthogonal frequency division multiplex (OFDM). This allows, on the one hand, to transmit a data-payload on top of the radar signal and to provide, on the other hand, a technique to implement interference mitigation mechanisms. Interference and its mitigation is already becoming an important issue with automotive radar because of the huge number of sensors already deployed and the much higher number envisaged to hit the roads in the near future. All these radars will of course influence each other leading to a decrease in the sensor performance. This puts a high pressure on the radars' developers to implement mitigation procedures. RTSs will have to implement interference sources in order to test these mitigations.
In the area of the antenna an increase of spatial resolution by applying multiple-in-multiple-out (MIMO) radars concept are investigated [19]. Those will allow to increase the angular resolution without increasing the complexity of the antenna hardware by the same factor. The massive signal processing necessary in MIMO radars to detect the targets must also be implemented in stimulation systems in order to generate those targets. The multiple channels between all antenna elements - both on the radar's transmit and receive side - have to be taken into account for a proper target stimulation.

Another issue is the processing of the so-called Micro-Doppler [19]. Micro-Doppler is the variation of the target's Doppler-frequency shift due to independent movement of components of the target, like the rotation of the tyres of a car or the motion of arm and legs of a pedestrian [20]. Those effects can be taken into account by updating the generation of the I/Q-components of the Doppler signal (compare Sect. 3.5).

All these trends will, of course, have a huge impact on a RTSsystem and may even require massive revision in the underlying structure. For the designer of a RTS it is therefore important to observe the developments in automotive radar very closely to be able to respond quickly to changes.

\section{Conclusion}

In this article we showed what is necessary to test ADAS functions on a ViL-testbed. After a general introduction of the involved concepts we focused on the automotive radar sensor and its stimulation. In a first step we derived the requirements and restrictions for the design of a RTS based on general properties of the sensors itself and their stimulation on a ViL testbed. Using this input we introduced the general structure of the hybrid analogue-digital RTS system. Thereafter, all corresponding components were presented, putting the emphasis on their seamless integration. In this context the design and implementation of this components were highlighted. Furthermore, it was shown how the requirements influenced the design and how the RTS based on this design is able to fulfil those requirements.

To validate the performance of the designed RTS measurements in the laboratory as well as on a roller test bench were performed. Despite of the industrial environment of the testbed we were able to properly stimulate the radar sensor including its object tracker.

Finally, we provided a short outlook on how automotive radar may evolve in the future. This summary indicated new challenges which

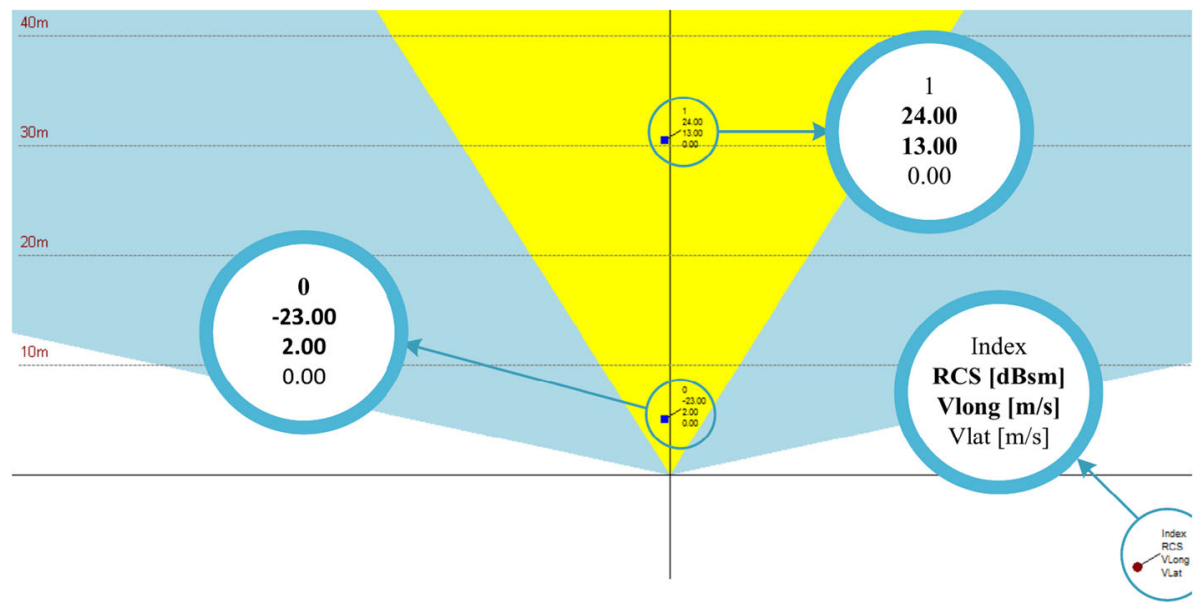

Fig. 11. The radar shows 2 stimulated targets 
must be handled by RTS systems to keep track with the upcoming developments.

\section{Acknowledgements}

Open access funding provided by Graz University of Technology. This work has been partially conducted within the ENABLE-S3 project which received funding from the ECSEL Joint Undertaking under grant agreement no 692455. This joint undertaking is supported by the European Union's Horizon 2020 Research and Innovation Programme and Austria, Denmark, Germany, Finland, Czech Republic, Italy, Spain, Portugal, Poland, Ireland, Belgium, France, Netherlands, United Kingdom, Slovakia, and Norway.

Additionally, this work was also funded by the Austrian Research Promotion Agency (FFG) under the research project GAZELE (No.: 848457).

Open Access This article is distributed under the terms of the Creative Commons Attribution 4.0 International License (http://creativecommons.org/ licenses/by/4.0/), which permits unrestricted use, distribution, and reproduction in any medium, provided you give appropriate credit to the original author(s) and the source, provide a link to the Creative Commons license, and indicate if changes were made.

\section{References}

1. Watzenig, D., Horn, M. (Eds.) (2017): Automated driving. Berlin: Springer. ISBN 9783-319-31895-0.

2. Schnieder, S. (2017): How to measure/calculate radar system MTBF? In European radar conference, workshop: automotive radar measurement solutions - for end-of-line purposes as well as in aftersales (pp. 1-24).

3. Maurer, M., Gerdes, J. C., Lenz, B., Winner, H. (2015): Autonomes Fahren: Technische, rechtliche und gesellschaftliche Aspekte. Berlin: Springer. ISBN 978-3-662-45854-9.

4. Koopman, P., Wagner, M. (2016): Challenges in autonomous vehicle testing and validation. In SAE world congress (pp. 1-10).

5. Gietelink, O., Ploeg, J., De Schutter, B., Verhaegen, M. (2006): Development of advanced driver assistance systems with vehicle hardware-in-the-loop simulations. Veh. Syst. Dyn., 44(7), 569-590.

\section{Authors}

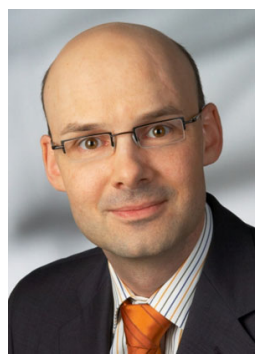

\section{Michael E. Gadringer}

received the Dipl.-Ing. and the Dr. techn. degrees from Vienna University of Technology, Austria, in 2002 and 2012, respectively. In July 2010 he changed to the Institute of $\mathrm{Mi}$ crowave and Photonic Engineering at Graz University of Technology, Austria. Since 2016, Michael Gadringer has been holding a tenure track research and teaching position at this institute. During his studies he was involved in the design of analogue and digital linearisation systems for power amplifiers and in the behavioral modeling of microwave circuits. In his current research activities he focuses on the design and linearisation of broadband microwave and mm-wave communication systems. Additionally, he is involved in planning and implementing complex measurements with an emphasis on calibration and deembedding techniques. Michael Gadringer has authored 13 journal and 32 conference papers. He fielded three patents and has coedited the book "RF Power Amplifier Behavioral Modeling", published by Cambridge University Press.
6. Versteegh, T. (2013): Youtube movie on VeHiL testing AEB Pre-Crash Braking System', Internet: https://www.youtube.com/watch?v=tWQPjXStSqs. Accessed: Feb. 25, 2018.

7. Grand cooperative driving challenge: Youtube movie demonstrating the concept (relative motion) of the VeHIL (Vehicle hardware-in-the-loop) facility. Internet: https://www.youtube.com/watch?v=HQT_8JAqrro Dec. 2009. Accessed: Feb. 25, 2018

8. Schyr, C., Brissard, A. (2016): DrivingCube-a novel concept for validation of powertrain and steering systems with automated driving functions. In International symposium on advanced vehicle control.

9. Gadringer, M., et al. (2017): Radar stimulation for a novel ADAS system. In Graz symposium VIRTUAL VEHICLE.

10. Maurer, L., Haider, G., Knapp, H. (2011): 77 GHz SiGe based bipolar transceivers for automotive radar applications - an industrial perspective. In IEEE 9th international new circuits and systems conference (pp. 257-260).

11. Meinl, F., Stolz, M., Kunert, M., Blume, H. (2017): An experimental high performance radar system for highly automated driving. In IEEE MTT-S international conference on microwaves for intelligent mobility, ICMIM (pp. 71-74).

12. Meinel, H. H., Dickmann, J. (2013): Automotive radar: from its origins to future directions. Microw. J., 9, 1-9.

13. Adamy, D. L. (2015): EW104: EW against a new generation of threats. Boston: Artech House. ISBN 978-1-60807-869-1.

14. Chrazanowski, E. J. (1990): Active radar electronic countermeasures. Boston: Artech House. ISBN 0-89006-290-0.

15. Lothes, R. N., Szymanski, M. B., Wiley, R. G. (1990): Radar vulnerability to jamming. Boston: Artech House. ISBN 0-89006-388-5.

16. VIRES Simulationstechnologie GmbH (2018): VTD—virtual test drive, Internet: https://vires. com/vtd-vires-virtual-test-drive. Accessed: Feb 23, 2018.

17. Maier, F. M. Makkapati, V. P., Horn, M. Environment perception simulation for radar stimulation in automated driving function testing. E\&l, Elektrotech. Inf.tech., https://doi.org/10.1007/s00502-018-0624-5.

18. ApisSys AV104 radar emitter-receiver DRFM. Internet: http://www.apissys.com/views/ media_produit/datasheets/6/Datasheet_AV104_Web-2.pdf. [Accessed: 26 Feb. 2018]

19. Kunert, M., Meinl, F. Stolz, M. (2016): Herausforderungen, Migrationspfade und Zukunftstechnologien auf dem Weg hin zum vollautomatisierten Fahren. VDI-Berichte, 2288, 169-184.

20. Chen, V. C. (2011): Micro-Doppler effect in radar. Boston: Artech House. ISBN 978-160807-057-2

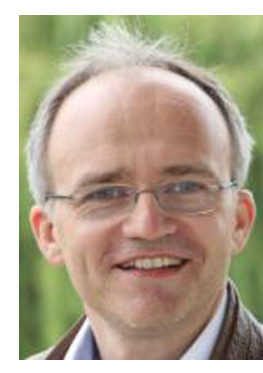

\section{Helmut Schreiber}

graduated to Dipl.-Ing. in electrical engineering in 1993 and Dr. techn. in 2007, both at Graz University of Technology, Austria. His professional career started in 1993 at the Institute of Communications and Wave Propagation. Since 2011 he has been projectsenior-scientist at the Institute of Microwave and Photonics Engineering of Graz University of Technology. His main research focus lies in the area of radar technology where he developed amongst others radar systems for the measurement of snow avalanches. Another major research topic is microwave propagation in which context he wrote multiple studies regarding the interference effects of structures like wind turbines or tower buildings on airtraffic-surveillance and navigation systems. 


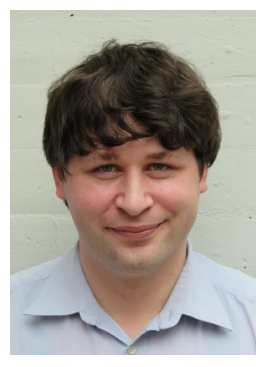

\section{Andreas Gruber}

studied information and computer engineering at Graz University of Technology, Austria with focus on telecommunication. Since January 2011 he has been employed at the Institute of Microwave and Photonic Engineering at Graz University of Technology, working as a system and hardware development engineer in the field of radar technology. He is member of IEEE and the Association of Old Crows.

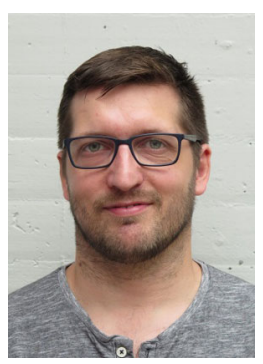

\section{Michael Vorderderfler}

studied information and computer engineering (telematics) at Graz University of Technology, Austria, and received his master's degree (Dipl.-Ing.) in 2016. During his studies, he focused on telecommunication and system-onchip design. In the course of his master's thesis he deepened his skills in microwave engineering and small antennas and gathered 2 years of practical experience in this field at semiconductor industry within his internship at Infineon Austria. Since 2016 he has been working as a scientific assistant at the Institute of Microwave and Photonic Engineering at Graz University of Technology. He started his Ph.D. study in summer 2016 with the focus on microwave circuit design, especially alternative frequency translation techniques at millimetre wave frequencies.

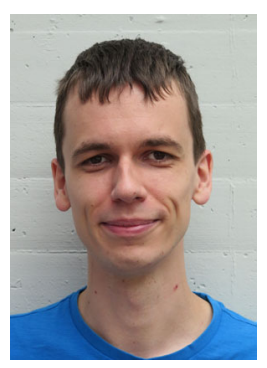

\section{Dominik Amschl}

finished the Higher Technical School for electrical engineering and started his professional career at the Institute of Microwave and Photonic Engineering at Graz University of Technology, Austria, in December 2010. He is the responsible engineer for microwave measurement tasks - especially on-wafer and antenna measurements. Furthermore, he participated in various projects as design and development engineer. Besides that, he started to study electrical engineering at Graz University of Technology.

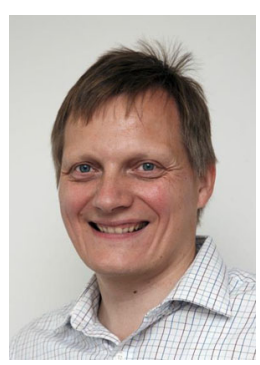

\section{Wolfgang Bösch}

joined the Graz University of Technology, Austria, in March 2010 to establish a new Institute for Microwave and Photonic Engineering. Prior he was the CTO of the Advanced Digital Institute in the UK, a not for profit organisation to promote research activities. Earlier he served as the Director of Business and Technology Integration for RFMD UK, and for almost ten years he was with Filtronic plc as CTO of Filtronic Integrated Products and the Director of the Global Technology Group. Before joining Filtronic, he held positions at the European Space Agency (ESA) working on amplifier linearization techniques, MPR-Teltech in Canada working on MMIC technology projects and the Corporate R\&D group of M/A-COM in Boston, USA, where he worked on advanced topologies for high efficiency power amplifiers. For four years he was with DaimlerChrysler Aerospace (now Airbus) in Germany working on T/R modules for airborne radar.
Professor Bösch received his engineering degrees from the Technical University of Vienna and Graz in Austria. He finalised his MBA with distinction at Bradford University School of Management, UK, in 2004. He is a Fellow of the IEEE and a Fellow of the IET. He has published more than 100 papers and holds 4 patents. He was a Non-Executive Director of Diamond Microwave Devices (DMD) and the Advanced Digital Institute (ADI). He is currently a Non-Executive Director of VIPER-RF company in the UK.

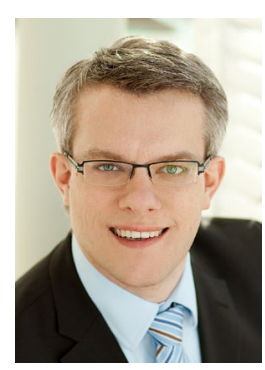

\section{Steffen Metzner}

is technology scout for the Instrumentation and Test Systems (ITS) division at AVL. He studied electrical engineering specialised in automation technologies at the University of Applied Science in Aachen, Germany, and control engineering at Coventry University, UK. Steffen joined AVL in 2007 as lead engineer responsible for the embedded systems software development process. In his current position he is responsible for the research projects regarding autonomous driving in his division.

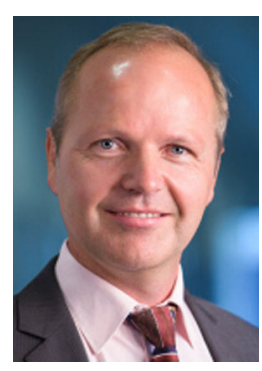

\section{Horst Pflügl}

received the Dipl.-Ing. degree from the Technical University of Graz in the field of electrical engineering/automation in 1995. He started his professional career at AVL as software developer for optimization software for engine control units and worked later as Head of Development. In this position he was responsible for the development and innovation strategy, he generated several patents as well as scientific publications and supported Master and Ph.D. students in this field of expertise. Since 2010 he has been in a central research department of $A V L$ responsible for the research program management of own and publicly funded research projects for the strategic business unit ITS (instrumentation and test systems), where he successfully set up and partly led important European research projects (CRYSTAL, ENABLE-S3, ASTERICS, OBELICS) in the area of vehicle development process, automated driving and electric driving.

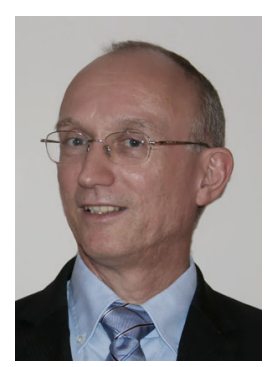

\section{Michael Paulweber}

received a master degree and a doctorate in control theory from Graz University of Technology, Austria, in 2008; he then added an Executive MBA degree at the St. Marys College of California, USA. After working at Graz University of Technology, he joined AVL List GesmbH and became head of development for the test system automation and control product-line. He gained expert knowledge in embedded software development. 2012 he was appointed director of global research and technology management in the AVL instrumentation and test system division. Michael Paulweber serves as vice president of the European Industry association ARTEMIS and is member of the European ECSEL steering board. He held lectures at a University of Applied Science as well as at Graz University of Technology 